\title{
RECOVERY FROM NATURAL AND MAN-MADE DISASTERS AS CAPABILITIES RESTORATION AND ENHANCEMENT
}

\author{
P. GARDONI ${ }^{1} \&$ C. MURPHY ${ }^{2}$ \\ ${ }^{1}$ Zachry Department of Civil Engineering, Texas A\&M University, USA. \\ ${ }^{2}$ Department of Philosophy, Texas A\&M University, USA.
}

\begin{abstract}
In the literature on the recovery of societies from natural disasters, a dominant theme is the importance of pursuing and achieving sustainable recovery. Sustainability implies that recovery efforts should aim to (re-) build, maintain, and, if possible, enhance the quality of life of members of the disaster-stricken community in the short and long term. In this paper, we propose a capabilities-based approach to recovery and argue that it provides important theoretical resources for better realizing this ideal of sustainability in practice. From a capabilities-based approach, the societal impact of a disaster is measured in terms of its impact on selected capabilities of individuals within society. Capabilities are constitutive elements of well-being and capture the valuable doings and beings individuals can achieve or become (e.g., being adequately nourished, and being sheltered). A proposed Disaster Impact Index (DII), we argue, can capture the societal impact of a disaster by measuring its impact on the well-being of individuals, as gauged by the changes in individuals' capabilities. We discuss how to measure this impact in practice. Also, a proposed Disaster Recovery Index (DRI) measures the current level of individuals' capabilities. It can provide important information on the degree to which capabilities have been restored and enhanced by comparing the DRI against a benchmark, or level of capabilities attainment, toward which recovery processes should strive. We argue that the DII and DRI provide critical information for policy- and decision-makers to use in order to practically implement the principles of sustainable recovery. Both can be used in the process of predisaster planning for recovery and in the period of recovery itself.

Keywords: capabilities, man-made disasters, natural disasters, sustainable recovery.
\end{abstract}

\section{INTRODUCTION}

Natural and man-made disasters can have enormous and devastating consequences. For example, in 2005 in the United States, Hurricane Katrina destroyed the Gulf Coast's highway infrastructure, damaged or destroyed 30 oil platforms, led to the closure of nine oil refineries [1], destroyed 1.3 million acres of forest lands [2], left hundreds of thousands of local residents unemployed [3] and displaced, and had an overall estimated economic impact of at least $\$ 150$ billion. As of April 2006, the United States government has requested \$105 billion for repairs and reconstruction in the region [4]. Given the enormous consequences natural and man-made disasters can have, it is imperative to be able to develop an effective strategy for recovering from natural disasters when they occur.

In the literature on the recovery of societies from natural disasters, the importance of achieving sustainable recovery is a dominant theme. In this paper we propose a capabilities-based approach to recovery and argue that it provides important resources for better realizing the idea of sustainability. Capabilities are constitutive elements of well-being. They describe the real, valuable possibilities open to a person. The proposed approach can be used both in the process of predisaster planning for recovery and in the period of recovery itself.

Section 2 describes the research into recovery that led to an emphasis on sustainability. Section 3 describes the idea of sustainability and the theoretical and practical issues associated with achieving sustainable recovery that the current literature on sustainability recognizes but does not sufficiently address. Section 4 describes the proposed capabilities-based approach, explaining its theoretical 


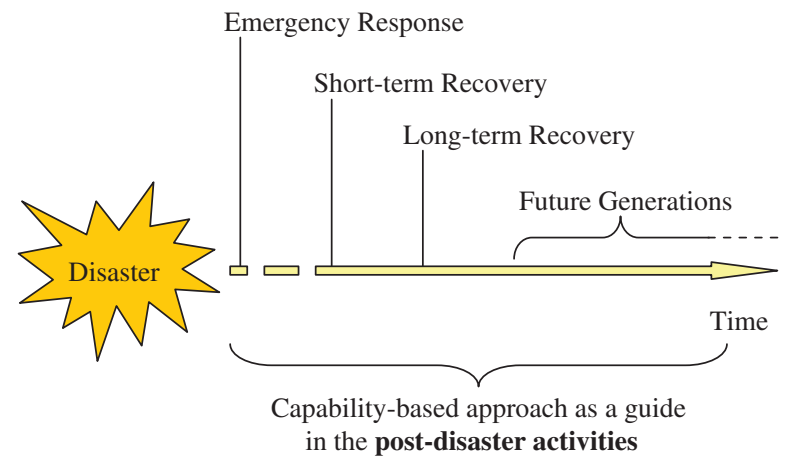

Figure 1: Phases post-disaster.

background, its use originally in development economics, and the application of this approach to risk analysis in our previous work. Section 5 outlines a capabilities-based approach to recovery. Section 6 outlines a method for practically implementing this approach. Section 7 discusses how the proposed approach addresses the issues described in the Section 3.

\section{OVERVIEW OF RESEARCH ON RECOVERY}

Historically, the recovery phase has been "the least investigated and most poorly understood of the four phases of a disaster - mitigation, preparedness, response and recovery" [5]. Broadly, the response phase covers the initial reaction to a disaster, whereas recovery deals with the short- and long-term reaction (Fig. 1). More specifically, the response phase begins with the search and rescue period, as part of the emergency response, and emphasizes "fulfilling the basic needs" of individuals. The recovery phase commences after the basic needs of individuals have been met and the immediate threat lessened. However, in the literature the precise demarcation of the recovery and response phases in the aftermath of a natural or man-made disaster and the precise definition of "recovery" remain the subject of debate ([6]; [7], pp. 230-231; [8]).

At its most abstract level, the goal of the recovery phase is the re-building of the community where the disaster occurred. More precise specifications of this general goal have been the subject of disagreement. According to one historically influential view, re-building entails re-creating the state of affairs that existed prior to the disaster ([7], p. 230). Recently, however, that goal has been questioned. Quarantelli, for example, argues that recovery implies "attempting to and/or bringing the post disaster situation to some level of acceptability. This may or may not be the same as the preimpact level" [6].

No matter how the goal of recovery is specified, the process of recovery is a complex and multifaceted process. A pressing dilemma facing decision-makers involved in the recovery process is how to balance achieving fast results with lasting and safe changes ([7], p. 233). A recovering community often would like, at the same time, to "(1) rapidly return to normal, (2) increase safety, and (3) improve the community" ([7], p. 233); not all of these desires may be satisfiable. Recovery also involves complicated questions regarding, for example, whether, and in what way, to rebuild homes, to repair and rebuild infrastructure, and to restore business [8].

Traditionally, research into recovery did not systematically address these theoretical issues. In fact, until recently there did not exist a "coherent body of knowledge" about how recovery should be planned. Disaster recovery resembled, in Mileti's view, "a set of processes in search of a policy" ([7], p. 235). 
Federal programs and guidelines for recovery often developed in response to particular events, rather than out of a comprehensive theoretical framework for recovery. At the federal level, this is partly explained by worries over the expenses of recovery programs, their effectiveness, the lack of data demonstrating the long-term impacts of disasters, and rising costs for disaster assistance ([7], p. 232).

Instead, research focused on identifying patterns in the recovery process among families, individuals, communities, or businesses, as well as obstacles to rapid recovery faced by these groups ([7], p. 230; [9-14]). There has thus been significant progress in understanding empirically trends in disaster vulnerability and recovery. The findings from this empirical research influenced the formulation of the principles of sustainable recovery, a coherent framework to guide recovery policy. Therefore, before turning to those principles, we discuss some of the patterns identified in these studies. Particular emphasis is placed on the role of socioeconomic status, race and ethnicity, and gender. As is clear in the next section, the principles of sustainable recovery are designed to address the problems identified and uncovered in empirical research.

Different degrees of vulnerability to disasters and rates of recovery exist among socioeconomic groups ([7], pp. 230-231). Poorer people tend to be more vulnerable in the sense that they are more likely to be a victim to a disaster and face greater difficulties in recovering from the losses due to a disaster [15]. Oliver-Smith [16] argues that disasters enhance pre-disaster inequalities. Boyce [17], in discussing the 1976 Earthquake in Guatemala, writes that it was classified as a "classquake" by researchers, because of its lopsided effects among the poor. Factors that contribute to this increased vulnerability include living in older and sub-standard structures, more densely populated areas, and landslide-susceptible ravines $[15,17]$. Inequality extends to the distribution of relief [18] and disastervulnerability recovery plans [17]. In practice, Boyce writes, "Richer individuals, groups and classes will get more out of the impure public good of disaster-vulnerability reduction than their poorer counterparts" [17].

Research also suggests that the impact of natural disasters affects minority populations and nonminority populations differentially. In the American context, race and ethnicity influence the process of recovery. In the long term, communities of color tend to face a greater drop in standard of living following a disaster $[9,15]$. Different attitudes toward the risks posed by disasters, disaster warning systems, and the reliability of the media exist among racial and ethnic groups [9]. In addition, minority groups tend to live in structures more susceptible to damage $[19,20]$. This, coupled with factors including lower incomes, higher unemployment, inadequate or no insurance, and reduced access to information, increase the difficulties minorities have in recovering [9, 20-22]. Race and ethnicity have influenced the quality of emergency relief provided by government services [18]. Language barriers, limited knowledge of the relief system, immigration status, and distrust in the authorities have impeded the accessibility of government relief $[9,19,20]$. Finally, research is also drawing attention to the importance of considering gender when examining the recovery process. Women often become primary caretakers for those affected by disasters, including the elderly, children, and injured. Further, gender roles and gender inequality in social, economic and political spheres are known to affect the accessibility and distribution of disaster relief [23, 24].

\section{SUSTAINABLE RECOVERY}

Responding to the absence of a comprehensive vision for recovery, Mileti [7] argued for a model of sustainable disaster recovery. The concept of sustainability became prominent in the late 1980s, especially through the World Commission on Environment and Development ([25], p. 188). In the context of development, sustainability captures the idea that efforts at development should not hamper the ability of future generations to fulfill their needs ([25], p. 188). Sustainability emphasizes inter- and intra-generational equity; adequate standards of living for everybody, and ecology ([7], p. 29). 
In the context of disaster recovery, sustainability implies that recovery efforts should aim to (re-) build, enhance, and then maintain the quality of life of members of the disaster-stricken community in the short and long term [26]. Below we discuss the five features of sustainable recovery and highlight what is still needed to realize them in practice.

\subsection{Restore, maintain and enhance quality of life}

Sustainable recovery emphasizes restoring, maintaining, and enhancing the quality of life of members of a disaster-stricken community [27]. Thus, the goal of sustainable recovery is not simply to re-create the conditions that existed before a disaster, but rather to work toward ensuring a decent quality of life both now and in the future (for the present and future generations).

Researchers advocating sustainable recovery emphasize that disasters create opportunities $[7,8,26,27]$. One opportunity is to mitigate the impact of future hazards ([5]; [7], pp. 237-238). Consequently, increasing numbers of researchers are calling for developing a pre-disaster plan within communities $[7,10,28]$. An additional opportunity is to correct problems that existed prior to the disaster. As Monday writes, "The concept of "sustainability" can provide an enlarged framework for examining potential mitigation measures - and any other community concerns - in a wider context" [26]. Examples of correcting pre-disaster problems include improving the available of affordable housing for the poor and improving traffic [5].

Two preconditions for realizing this goal of restoring, enhancing, and maintaining the quality of life of members of a disaster-stricken community are the following: (1) clarifying the meaning of the "standard of living," and (2) having a method for measuring or assessing the standard of living. Many components and factors affect and define the quality of life of individuals. Advocates of sustainable recovery recognize that quality of life is a complex and multifaceted concept. The list of factors that influence the quality of life cover economic, social, educational, and ecological aspects. However, there are practical challenges in implementing sustainable recovery because assessing the quality of life in a community is complicated. There remains no articulated and principled basis for the inclusion of the different components listed above. That is, there are no principled grounds for including or excluding a potentially significant or defining aspect in the assessment of the standard of living. In addition, the practical implementability of the sustainable recovery approach depends on our ability to assess the current quality of life of members of a community as well as changes to that standard. However, a systematic method for gauging the standard of living of a society is currently missing.

\subsection{Promote social equity (intra-generational justice within a society)}

According to advocates, sustainable recovery should be equitable ([27]; [29], p. 127). Resources for recovery and the opportunities recovery processes create should be available to all members of a community ([25], p. 188). Indeed, some authors assert a basic (and egalitarian) right to a safe and clean environment [17]. The implications of a commitment to egalitarian recovery include treating mortality of all individuals as equally significant [17].

To determine whether recovery is equitable, it is necessary to have a method for ascertaining the recovery of disparate social groups. Information on rates of progress among socioeconomic, racial, and ethnic groups, for example, can draw attention to inequalities when they exist. This measurement of equity must be sensitive to the problems with relief distribution of the past. It must, for example, look beyond simply how much aid is available to groups of individuals within society and consider whether that aid is in practice accessible, given, for example, language and cultural barriers. 
The examination of equity should also factor in differences in the effect or impact in recovery of distributed aids and future vulnerability [5, 28, 30]. Similarly, it must factor in the non-uniform spatial distribution of people and population density. In Boyce's words, "There is a difference between saying that each individual has an equal right to risk mitigation and saying that the weight on each individual's risk should be equal" [17].

\subsection{Promote inter-generational justice}

Recovery potentially impacts the welfare of both current generations and future generations. One goal of sustainable recovery is to respect and foster intergenerational equity ([7]; [25], p. 188). That is, recovery should not undermine the ability of future generations to achieve or enhance their well-being.

To determine whether recovery processes achieve this goal, it is important to be able to update recovery plans and forecast likely future well-being. The importance of updating occurs not only as the recovery phase in the aftermath of an actual disaster proceeds but also in the recovery planning phase. Such forecasting exercises must take into account two sources of uncertainty. First, there is uncertainty surrounding the likely impact of recovery policies. The recovery process impacts longterm well-being and the well-being of future generations. Thus, there needs to be a method for updating in particular contexts how the current state of recovery will impact well-being in the future. "No emergency plan is likely to remain fully operational and completely efficient over time unless it is updated" ([29], p. 131). In the context of emergency planning and preparation, Alexander ([29], p. 131) suggests adjusting recovery plans as conditions change, including available emergency resource levels, technological changes, and increased information about a hazard. To some extent, this practice of updating is currently occurring in the business community through business continuity planning, which includes annual testing and updating of post-disaster recovery plans [31, 32].

Second, there is the more general uncertainty surrounding the conditions necessary for sustaining a desired level of well-being. As Goodin [33] writes, "we can never be quite sure what consumption levels might actually be sustainable into the indefinite future." When resources become scarce, prices increase; this creates an incentive to search for new supplies or new substitutes. "Allowing for substitutability, future generations might be as well off as present ones in terms of all the functional tasks performed, albeit using a different array of material items to perform those functions" [33]. Thus, it is difficult to determine what is fair to ensure that future generations have simply by considering what resources present generations have available. Similarly, future generations might have new technologies or engineering solutions that make current ones obsolete and therefore saving some resources or designing solutions with future generations in mind might be a waste of resources. Finally, as current and future generations acquire more knowledge, current solution strategies might turn out to be even deleterious (an example is the use of cancerogenous materials in construction that contains asbestos).

\subsection{Address environmental concerns}

Sustainable recovery implies a commitment to ensuring that ecosystems are able to renew themselves and to viewing members of a community as co-existing with the natural ecosystem. It tries to avert the degradation of natural systems and to replace practices which threaten the sustainability of ecosystems. To the extent that an ecosystem is damaged, sustainable recovery would suggest working to restore or rehabilitate that system. Similarly, recovery tries to reduce future environmental vulnerability and promote sustainable changes $[34,35]$. 
Members of a society can become motivated by these environmental concerns through understanding that a flourishing natural ecosystem is also an important element for the well-being of individuals, for the sake of protecting nature itself, or a combination thereof. However, a method is needed to account for the environmental concerns in the overall well-being of individuals and to gauge the impact on the well-being from a flourishing natural ecosystem compared to other influencing factors that also promote well-being.

\subsection{Facilitate public participation}

The complex and lengthy recovery process involves many organizations and individuals, including local, federal, and state governments, businesses, politicians, and community activists [8]. Often members of the international community and international organizations are also involved. The function played by organizations differs; some organizations are regulatory, others financial (i.e., provide support) [8]. Increasingly, research recognizes the influence of social norms and public perceptions in the process ([7], p. 239; [36]). This has led to calls for greater participation by members of the disaster-stricken community. The benefits of such processes include having a community feel greater "ownership" over the recovery process, promote a sense of community, the production of new ideas, and an increased likelihood that recovery decisions will be viewed as legitimate. Lack of public participation can inhibit the promotion of social equity. Frequently those segments of society that are most marginalized and have less trust in the authorities are then less likely to participate in the recovery and to benefit from the recovery aids.

For participation to be helpful and productive, it is essential that the public be informed. One challenge to ensuring public participation is determining how to effectively communicate and disseminate the necessary information. Equally important is finding a way to balance the long-term goals of sustainable recovery with the desire of disaster-stricken communities to have life return to normal as quickly as possible [10]. "The pressure to move quickly can result in hasty decisions not fully thought through" [10]. It is important to consider the conditions that are likely to lead to less hasty decision-making, should public participation be encouraged.

One final issue to consider is how to encourage dialog among the various actors working for sustainable societies in different contexts [37]. Berke laments the lack of interaction and communication between advocates of sustainable development and risk analysts. He argues that disasters are most often conceived of as a nuisance that needs to be addressed using more than short-term options. Sustainable recovery, especially its aim of mitigating hazards, will be more effectively realized if the processes that occur in conjunction with recovery efforts, in particular development efforts, incorporate the importance of risk mitigation into their processes.

\section{A CAPABILITIES-BASED APPROACH}

\subsection{Background}

Sen [38-42] and Nussbaum [43, 44] first articulated a capabilities-based approach in the field of development economics and policy. A primary issue in that field is how to assess the level of development of societies. From a capabilities-based approach, the level of development in a society is a function of the level of well-being or standard of living of individuals within that society. A capabilities-based approach gauges the standard of living of individuals by deter mining their capabilities, or "the ability of people to lead the kind of life they have reason to value" [45]. 
Capabilities are defined in terms of functionings or "valuable acts or [...] states of being" ([41], p. 30). Functionings denote what an individual may do or become in life that is of value. Examples of functionings are being alive, being healthy, and being sheltered. Capabilities represent the actual ability to achieve their corresponding functioning and thus are positive freedoms. An underlying assumption of a capabilities-based approach is that freedom from external interference, or negative freedom, is not sufficient for individuals to be actually free to realize valuable functionings.

Capabilities are distinct from other metrics of well-being, including utility or resource-based metrics. Utilities try to capture the mental satisfaction or happiness of individuals ([41], p. 58). Utilities are determined in practice by considering individual's choices or preferences. $A$ has more utility than $B$ if it is preferred to or would be chosen over $B$. Capabilities do not emphasize mental satisfaction, but the real opportunities individuals have. Capabilities have the benefit of avoiding the problem of adaptive preferences. Adaptive preferences refer to the phenomenon of individuals shaping their expectations and preferences by what is realistic, given their circumstances. An individual living in extreme poverty may develop limited expectations, all of which are satisfied. From the utilitarian perspective, that individual may be said to be well-off, despite being objectively deprived. From a capabilities-based approach, an assessment of well-being would take into account this poverty and consider how it negatively impacts what one is able to do or achieve, and consequently one's well-being.

Capabilities are also distinct from resources. A common resource-based metric of well-being is primary goods or all-purpose means that enable individuals to pursue their goals and aims. Examples of primary goods include rights, income, and opportunities. Looking at the amount of resources an individual has is insufficient to gauge that individual's well-being because of what Sen labels the interpersonal conversation rate ([41], pp. 70-75). With identical amounts of resources, different individuals may not be able to achieve identical goals. Prior recovery and relief efforts discussed above demonstrate that setting aside a certain amount of relief resources for individuals is not enough to guarantee that individuals will actually be able to avail themselves of those resources in practice. Language barriers, distrust of government officials, lack of knowledge of the relief system all can undermine the ability of individuals to access relief to which they are entitled in principle. Thus looking at the resources available in principle to individuals is not sufficient for knowing how well individuals actually are. "(A)s human beings differ in their abilities to transform resources into useful functionings, then a resourcist approach cannot tell us who is truly well or badly off" [46]. Capabilities, on the other hand, concentrate on what individuals are able to do with resources and measures well-being in a way that takes into consideration how these various factors influence individuals' ability to utilize resources. In addition, capabilities are constitutive components of well-being and valuable in themselves. Resources and primary goods, however, measure important means to freedom, but not well-being directly [42].

\subsubsection{Development economics and policy}

A capabilities-based approach is currently used by the United Nations (UN) and development agencies to assess the development of societies. Annually, the UN publishes the Human Development Report (HDR), which provides a picture of the level of development of counties. A primary objective when the first HDR was published in 1990 was to center economic debate, policy, and advocacy on people's well-being and to assess well-being without looking solely at income.

The HDR uses the Human Development Index (HDI) to determine the level of development. The HDI assesses development based on the level of achievement of three primary capabilities (the capability to live a long and healthy life, the opportunity for being knowledgeable, and the capability of having a decent standard of living). In practice, indicators are employed to measure the level of 
capabilities attainment, since capabilities are not directly quantifiable. Each indicator serves as a gauge for a specific capability [47, 48]. Leading scholars and practitioners in the field of development economics contribute to the HDR. Currently, four additional indices are also used: the Human Development Index, the Gender-related Development Index, the Gender Empowerment Measure, and the Human Poverty Index.

4.1.2 Risk analysis, public policy, and resource allocation in hazard mitigation

In this paper we describe a capabilities-based approach to the measurement of the actual societal impact of a disaster in the immediate, short, and long term. This information is critical for evaluating and implementing recovery plans. This approach values protecting and promoting individuals' capabilities. It also builds on our previous work developing a capabilities-based approach to risk analysis related to natural hazards and disasters.

In Murphy and Gardoni [49] and Gardoni and Murphy [50], we propose a capabilities-based approach as the theoretical foundation for identifying and quantifying the potential societal impact of natural hazards in a more satisfactory way. In a capabilities-based approach, the potential benefits and losses due to a hazard are measured and compared in a uniform way by using individual capabilities (specified functionings individuals are able, still able, or unable to achieve) as a metric. In a capabilities-based approach, risk refers to the probability that individuals' capabilities might be reduced due to a hazard. In Gardoni and Murphy [50] we develop a methodology to quantify the expected societal impact of a hazard, or the actual societal impact of a disaster in order to practically implement the proposed capabilities-based approach.

In Murphy and Gardoni [51], we argue that an overarching goal of policy makers dealing with hazard mitigation should be the mitigation of the impact of natural hazards on individuals' capabilities. For this to be possible, policy makers need a method for identifying when mitigation is necessary and which mitigation and resource allocation policies to pursue. Murphy and Gardoni [51] describe our proposed capabilities-based approach to public policy and resource allocation. The first step in this method is to consider whether the risk posed by a natural hazard is either acceptable or tolerable (see [52] for a discussion of how to determine acceptable or tolerable risks). In general, priority should be given to addressing or mitigating the impact of intolerable risks first, followed by addressing the tolerable but unacceptable risks. The same principle for prioritizing which risks to address can be used to determine which mitigation strategies for intolerable and unacceptable risks are in practice viable options. Viable options are those that are likely to successfully bring individuals above the acceptable or tolerable levels. From a capabilities-based approach, viable mitigation strategies should be chosen on the basis of their likely affectability. That is, we should compare the expected dollar per unit of change in the societal impact of a hazard that various mitigation strategies might have. As noted earlier, such change can be achieved by reducing either the probability of occurrence of a hazard and/or by limiting its impacts. This is done to maximize the return on the investment made using public resources.

\section{RECOVERY AS CAPABILITIES RESTORATION AND ENHANCEMENT}

In this section we present a capabilities-based approach to recovery. Capabilities are constitutive elements of well-being. Thus, a capabilities-based approach focuses directly on the elements of interest in the recovery process. We first discuss the general framework for assessing the impact, tracking progress in recovery over time, and evaluating the effectiveness of recovery processes. We also consider how issues of inter- and intra-generational justice can be accounted for. We then describe in detail how a capabilities-based approach can be practically implemented. Finally, we explain the benefits of conceptualizing sustainable recovery in terms of capabilities and how the proposed approach helps implement the five features of sustainable recovery. 
From a capabilities-based approach, the societal impact of a disaster is measured in terms of its impact on selected individuals' capabilities within society. As its original application in development economics and policy shows, a capabilities-based approach is ideally suited for gauging how well off individuals in a disaster-stricken society are. A Disaster Impact Index (DII) can capture the societal impact of a disaster by measuring the change in well-being. Specifically, the DII can be computed as the difference between the value of a society's level of well-being, achieved before a disaster strikes and the value at any time $t$ after the occurrence of the disaster. So the DII can be written as $\operatorname{DII}(t)=\operatorname{DRI}\left(0^{-}\right)-\operatorname{DRI}(t)$, where $\operatorname{DRI}\left(0^{-}\right)$is the Disaster Recovery Index (DRI) immediately before a disaster strikes and represents the level of well-being of the society pre-disaster and DRI $(t)$ represents the level of well-being of the society at time $t$ after a disaster strikes. When $t$ is within the emergency phase, DII $(t)$ provides the impact in the immediate aftermath of a disaster. As $t$ enters in the short-/long-term phases of recovery, $\mathrm{DII}(t)$ shows the amelioration of the societal conditions post-disaster. Below we discuss how to measure this impact in practice.

The focus on capabilities is maintained when evaluating the level of recovery a disaster-stricken community has achieved. A process of recovery from natural and man-made hazards has many steps that can be taken over time. A capabilities-based approach can gauge the extent of recovery and the progress made over time. This is assessed based on the level of individuals' capabilities at given moments in time of the recovery process. The DRI measures the current level of individuals' capabilities. Examples of capabilities to consider include the capability to be adequately nourished, have adequate and permanent shelter, engage in interaction with others, and be mobile. The DRI provides information on the degree to which capabilities have been restored and enhanced by comparing the DRI against a benchmark, or level of capabilities attainment toward which recovery processes should strive. That benchmark does not need to be identical to the level of the capabilities attainment before the disaster. Rather, in the spirit of the commitment of sustainable recovery, the level of attainment should be specified in terms of the level that we ideally want to see all citizens achieve.

We can assess the effectiveness of recovery policies that have been implemented or are being considered. To make this evaluation for already implemented policies, we can look at the change or improvement effected by the policy as measured by the change in DRI. We can predict the likely improvement or effectiveness of a policy by predicting the likely DRI. Such predictions can be based on information provided by engineering or sociological models or by looking at values of the DRI in similar situations. The change over time of the well-being of individuals can be monitored to assess the recovery process, to see which interventions are most effective, and to avoid inter-generational injustice. After quantifying the expected DRI associated with a decisions or resource-allocations or recovery strategy, one can compare the associated DRIs also considering their associated costs. This provides a useful tool for decision-makers in the process of resource allocation and policy making. The relative effectiveness of various policies can be measured by predicting their DRI if implemented, when policy options are being compared to implement at the same point (because the starting point will be the same in each case and so the DRI will capture the relative difference). When assessing the actual difference between DRI $\left(t_{2}\right)$ (after the policy is implemented) minus DRI $\left(t_{1}\right)$ (before the policy is implemented) where $t_{2} \geq t_{1}$. (Note that this is the same as doing the $\operatorname{DII}\left(t_{1}\right)$-DII $\left(t_{2}\right)$, since $\operatorname{DRI}(0)$ cancels out.) Finally, in recent years, pre-impact recovery planning [28] has been promoted by researchers and practitioners with the aim of reducing or minimizing the impact of potential, future natural hazards. The proposed approach can be used to assess the effectiveness of alternative pre-impact recovery planning over time, by comparing the DII of different cities or regions affected by natural disasters that did not implement or implemented different pre-impact recovery plans. 


\section{PRACTICAL IMPLEMENTATION}

In this section, we discuss how the proposed approach can be implemented in practice. We focus on the steps that need to be taken to construct a given DRI, and, by extension, the DII, in order to assess the societal impact of a hazard, track the progress of recovery over time, and assess the impact of policies over time. The DRI is modeled on the HDI in the context of development. The methodology for constructing the DRI is modeled on that used to construct the HDI.

\subsection{Selection of capabilities}

The first step in a capabilities-based approach to recovery is to identify the capabilities to take into account when assessing the societal impact of a disaster both initially and over time. There are four criteria selected capabilities should fulfill.

1. Relevance: Selected capabilities should have a direct link to our general purpose in recovery efforts. In Sen's words, "[t]he focus has to be on the underlying concerns and values, in terms of which some definable capabilities may be important and others quite trivial and negligible" [39].

2. Importance: Selected capabilities need to be significant, in the sense of capturing an effect of a disaster that should be factored into the calculation of the societal impact. Chosen capabilities should be appropriately the subject of recovery planning and policy. Given that public resources are limited, the consequences of a disaster that are addressed using public resources should be of significant value.

3. Influenceability: In the recovery process it is essential to allocate limited resources in the most effective manner. Influenceability means that those capabilities that are selected to assess the societal impact of a disaster can be tangibly affected by resource allocations and recovery actions.

4. Practical implementability and accuracy: The practical value of the capabilities-based approach depends on our ability to assess the impact of a disaster on capabilities. This in turn depends on information about changes in capabilities being accessible. Equally important, to ensure that the assessed impact is accurate it is important to include all the relevant information. Two conditions help to ensure the practical implementability and accuracy of this approach. First, the fewest possible capabilities should be chosen (capabilities parsimoniety). This reduces the amount of data needed to be acquired in the aftermath of a disaster to measure that disaster's impact and enhances the framework's practicability. Second, the capabilities chosen should each provide unique sources of information about the change in well-being of individuals not captured by the other chosen capabilities (capabilities orthogonality).

\subsection{Identification of indicators}

\subsubsection{Selection criteria}

Capabilities cannot be directly measured or quantified. Therefore, it is necessary to have indicators for each capability. For example, the capability to have adequate and permanent shelter, an indicator could be the number of persons left homeless due to a hazard. A potential indicator for the capability to engage in interaction with others is the number of individuals unemployed due to a disaster. These indicators signify or provide a picture of the level of or changes in individuals' attainment of capabilities. The societal impact of a disaster should be assessed based on how it affects individuals' 
capabilities. Given the critical role indicators play in quantifying this impact of a disaster, it is important to be sure appropriate and accurate indicators are selected. Indicators should meet two general criteria to ensure they are appropriate and accurate measures of specific capabilities.

1. Be representative of the corresponding capability: An indicator should correlate with the capability it signifies. If it does not correlate, measuring an indicator provides irrelevant information, given hat is supposed to be gauged. Such correlation can be explored by looking at the value of an indicator and comparing that with the level of achievement of the corresponding capability, which has to be assessed through a qualitative analysis. Appropriate indicators may be context dependent or hazard dependent.

2. Be intuitively plausible: There may be indicators that can be shown to be correlated with a given capability, but the correlation cannot be intuitively justified or explained. These indicators could be used to assess the societal impact of a hazard. However, their use would hamper the general communicability of and trust in the results from the approach, given that the connection between the information and the level of well-being is not obvious or understandable. To ensure that the capabilities-based approach is transparent and easily communicable and understandable to the public and policy makers, it is better to have indicators which intuitively show why the indicator tracks the capability in which we are interested in addition to being shown to be correlated. Engineering practice can serve as a starting point for selecting potential indicators. Information about the consequences of disasters gathered by engineers seems intuitively to capture important effects of such disasters. In implementing a capabilities-based approach the task is to determine for which capabilities such indicators are relevant.

\subsection{Scaling of indicators: indicator indices}

Each indicator provides a measure of a distinct aspect of the well-being of individuals. To make the information from different indicators comparable, the indicators must be normalized (or scaled) into a common metric. Through the scaling process, indicators can be combined, creating a comprehensive composite index. As in constructing the HDI, in developing the DII indicators are normalized to create dimensionless indices that range from 0 to 1 . A 0 value of the index indicates that the disaster had no impact on the corresponding indicator. Conversely, a value of 1 indicates that the disaster had the maximum foreseeable impact on the corresponding indicator.

\subsection{Weighting the indicator indices: Disaster Index}

The Disaster Index (DI) is a normalized composite measure of the net overall impact of a disaster. A DI is computed as the potentially weighted average of the IIs. As when constructing the HDI, the IIs should, in our view, have equal weight [47]. This is because the value of each capability is incommensurable. An excessive level of one capability cannot compensate for the absence or lack of another capability. For example, no level of command over resources can make up for the lack of physical and mental health.

\subsection{Putting the Disaster Index in context: Disaster Impact Index}

While the DI provides a picture of the net overall impact of a disaster, the actual impact on an individual in a society will vary based on the size of the society. For example, the impact of 
3000 fatalities in El Salvador will be different from the impact in China. Consequently, to determine the impact of a hazard on individuals, on average, the DI should be divided by the number of individuals in the society, defining the DII. For a given DI, a larger society results in a smaller average impact on an individual since more people share in the impact and there are likely to be more resources to draw on in the process of recovering from a disaster. Conversely, for the same $D I$, in a smaller society the average impact on an individual is larger since fewer people share in the impact and there are likely to be fewer resources available for the process of recovery. By looking at the average impact of a hazard on individuals, the DII makes that impact more meaningful.

\subsection{Updating information on recovery in the short- and long-term}

It may be necessary to update the indicators used to gauge selected capabilities in order to measure accurately the degree of recovery a society has achieved and to assess the effectiveness of recovery policies. The indicators used to assess the impact of a disaster in the immediate aftermath may need to be supplemented with additional indicators more applicable to the long- and short-term recovery phases. There are two reasons why it may be necessary to add more indicators. First, while initial, easily measured and readily available indicators may be useful for getting a basic or coarse sense of the impact of a hazard in the emergency phase, over time such coarse indicators may offer a mistaken picture of recovery or the impact on a community. Measuring recovery or the impact on the base of coarse or more basic indicators may mistakenly suggest that a community has completely recovered or is no longer impacted by a disaster, when subtler impacts on a disaster-stricken community remain and intuitively it seems it has not.

For example, an estimate of the number of people missing can be used to assess the capability to live to the normal end of life. This indicator would be appropriate to get an initial basic sense of the impact of a disaster on a community and can provide important information for emergency response personnel to consider. Other, more refined indicators of this same capability, for example, the number of fatalities and number of injuries, may take longer to assess, because of the time required to get the relevant data. It may be possible to use these indicators to assess the impact of a disaster in the short and long term or to track recovery.

Similarly, a DRI that gauged the elementary achievement of specified capabilities will not be very useful in assessing the improvement in capabilities within society in the short- and long-term recovery phases. To illustrate, consider the capability to be sheltered. An initial indicator of this capability could be whether or not an individual had shelter. The percentage of a population left homeless as a result of a disaster could offer an initial assessment of the general attainment of the capability to be sheltered. In the long term, however, the capability may not fully have been restored or a community recovered, despite the fact that few or none are homeless. A significant percentage of housing may still be temporary. A later additional indicator of the same capability could be represented by the percentage of the population with a permanent residence.

The second reason for updating indicators over time is to account for the impact of technological or social change on the realization of capabilities, for present and future generations. For example, a disaster before the advent of cellular phones could have disrupted the ability of individuals to communicate with other cities or regions. So assessing the damage to the landlines could have helped gauge the capability to communicate and could have been selected as an indicator. However, with the advent and diffuse use of cellular phones, assessing the damage to the landlines no longer provides a reliable assessment of the ability of individuals to communicate. Additional or different indicators need to be included. The ability to communicate might be better assessed by considering the damage to the wireless communication network. 


\section{BENEFITS OF A CAPABILITIES-BASED APPROACH TO RECOVERY}

In this section we discuss how the proposed approach can promote the five principles of sustainable recovery discussed in Section 3. We also discuss additional benefits of the Capabilities-based Approach to recovery.

\subsection{Restore, maintain and enhance quality of life}

A capabilities-based approach shifts the focus of recovery officials to ensuring capabilities are protected, restored, and enhanced. One main goal of sustainable recovery is to enhance and restore the quality of life of individuals. The capabilities-based approach offers a tool to assess whether this is in fact occurring. The DII and DRI help to quantify the impact of a disaster on the well-being of individuals and to assess the degree to which well-being has been restored.

The capabilities-based approach also has the resources for motivating recovery officials to ask what victims of a natural hazard need in order to be in a position to benefit in practice from relief provided by federal and local governments. Instead of focusing strictly on ensuring relief resources are set aside, a capabilities-based approach would consider whether information programs should be established to alleviate concerns and fears of those suspicious of government agencies. Ensuring that language barriers are sufficiently addressed would be another recommendation. Guiding these questions is an over-arching concern with ensuring that individuals are able to function fully.

\subsection{Promote social equity (intra-generational justice)}

A method of disaggregation can be used to determine the differential impact of a disaster among socioeconomic, racial, geographic, and occupational groups. The method consists in assessing the DII for a selected sub-group of the population, generating a set of DIIs, one for each sub-group. Comparing the DIIs provides an insight into potential intra-generational injustices. This method of disaggregation enables us to ascertain whether all groups within society are making progress toward the level of capability attainment that is the goal of recovery process, which is an important goal of sustainable recovery. It thus can be used to assess intra-generational inequalities and to guide effective interventions that provide a uniform well-being. This same method of disaggregation is used by the United Nations to gauge the level of development of groups within a society. Disaggregation can focus on geographic, ethnic, and gender differences [45].

\subsection{Promote inter-generational justice}

It is possible to use the above methodology to calculate the impact of policies on the well-being that accounts for the impact on the well-being of future generations (predicted) as well as present generations. Future generations can be included in the forecasting of the impact of policies that are being considered, as well as included as likely impacts in actual implemented policies. The impact on future generations can also be included in the determination of the DII and the DRI at any given interval. Given that the well-being of future generations is predicted, we need to include the probability that the anticipated indicator level will be realized. The further into the future we go, the less reliable the predictions become. The discussion on updating above lists two important factors for this diminished reliability. Technological innovations in the future may alter the impact of policies in the present, for example. Thus, it may be necessary to attribute less weight to the predictions, though not less weight to the well-being of future generations. Whether to assign the same weight to 
the predicted DII for future generations or, more generally, how to account for this diminished predictive reliability, is a decision that decision-makers and the public will have to make.

\subsection{Address environmental concerns}

From a capabilities-based approach it is possible to include the impact of a disaster on the environment. Indeed, one capability Nussbaum defends is the capability to live "with concern for and in relation to the world of nature and animals" [43]. Permanent environmental damage can undermine this capability. Implicit in this capability is the assumption that the natural environment plays a critical role in the achievement of individual well-being.

\subsection{Facilitate public participation}

An important goal of sustainable recovery is to increase the participation of the public. For public participation to be maximally beneficial, it is important that the public be informed about the implications of various policies considered, the impact of a disaster, and progress toward recovery. Creating and maintaining an informed public requires relevant information be presented in understandable terms. The transparency of the proposed approach will promote the participation of victims of a hazard. This approach is transparent in that the value judgments underlying its measurement of the impact of a disaster and evaluation of policy options, actually taken or under consideration, are explicit. Protecting individuals' capabilities and bringing all groups within society to the desired capabilities levels is the overarching goal. This transparency makes it easier for the public to discuss and decide whether these are value judgments that they want to endorse. Similarly, the intuitively plausible measures used to assess well-being makes the results of policy evaluations and judgments about the degree of recovery more understandable.

\subsection{Encourage sustainability in development}

A final, additional benefit of our capabilities-based approach is that it offers a common approach to both the process of recovery and the process of development. This can encourage or facilitate communication among members of the recovery and development communities. As noted above, recovery often poses opportunities, an important one of which is for development. Using the same tools can facilitate the promotion of development through recovery. This is significant because the impact of a given hazard is more significant on developing countries. Developing countries are often more vulnerable to natural and man-made hazards, prompting the issue of development through recovery.

\section{CONCLUSIONS}

In this paper, we have proposed a capabilities-based approach to recovery and argued that it provides important theoretical resources for better realizing this ideal of sustainability in practice. A capabilitiesbased approach assesses the societal impact of a disaster based on its impact on selected capabilities of individuals within society; capabilities are constitutive elements of well-being. We discussed a DII which, we argued, can measure the societal impact of a disaster, based on the changes in individuals' capabilities. Also, a proposed DRI can measures the current level of individuals' capabilities, providing important information on the degree to which capabilities have been restored and enhanced. We have argued that the DII and DRI provide critical information for policy- and decision-makers to use in order to practically implement the principles of sustainable recovery. Both can be used in the process of predisaster planning for recovery and in the period of recovery itself. 


\section{ACKNOWLEDGMENT}

The authors thank Mrs. Kathleen Murphy for helpful suggestions.

\section{REFERENCES}

[1] United States Department of Commerce, Hurricane Katrina Service Assessment Report, Retrieved on 2006-07-14, 2006.

[2] Sheikh, P.A., The Impact of Hurricane Katrina on Biological Resources, Congressional Research Service, Retrieved on 2006-06-05, 2006.

[3] Burton, M.L. \& Hicks, M.J., Hurricane Katrina: Preliminary Estimates of Commercial and Public Sector Damages, Marshall University: Center for Business and Economic Research. Retrieved on 2006-06-05, 2005.

[4] St. Onge, J. \& Epstein, V., Ex-chief says FEMA Readiness Even Worse. Boston.com, Retrieved on 2006-06-05, April 1, 2006.

[5] Berke, P.R., Karetz, J. \& Wenger, D., Recovery after disaster: achieving sustainable development, mitigation, and equity. Disasters, 17(2), pp. 93-109, 1993.

[6] Quarantelli, E.L., The Disaster Recovery Process: What we Know and Do Not Know From Research, Preliminary Paper \#286, University of Delaware Disaster Research Center, 1999.

[7] Mileti, D.S., Disasters by Design: A Reassessment of Natural Hazards in the United States, Joseph Henry Press: Washington, D.C., 1999.

[8] Haddow, G.D. \& Bullock, J.A., Introduction to Emergency Management, ButterworthHeinemann, p. 95, 2003.

[9] Fothergill, A., Maestas, E.G.M. \& Darlington, J.D., Race, ethnicity and disasters in the United States: a review of the literature. Disasters, 23(2), pp. 156-173, 1999.

[10] Petterson, J., A Review of the Literature and Programs on Local Recovery from Disaster, Working Paper \#102, Natural Hazards Research and Applications Information Center, Institute of Behavioral Science, University of Colorado, www.riskinstitute.org, 1999.

[11] Dahlhamer, J. \& D’Souza, M., Determinants of business disaster preparedness. International Journal of Mass Emergency and Disasters 15, pp. 265-281, 1997.

[12] Tierney, K., What are the likely categories of loss and damage? The Economic Consequences of a Catastrophic Earthquake: Proceedings of a Forum, National Academy Press: Washington, D.C., pp. 77-82, 1992.

[13] Tierney, K., Societal impacts. The Northridge, California Earthquake of January 17, 1994: General Reconnaissance Report, ed. J. Goltz, National Center for Earthquake Engineering Research: Buffalo, NY, pp. 7:1-7:10, 1994.

[14] Nigg, J., Anticipated business disruption effects due to earthquake-induced lifeline interruptions. Post-Earthquake Rehabilitation and Reconstruction, eds F. Cheng \& Y. Wang, Pergamon, pp. 47-57, 1996.

[15] Miller, K. \& Nigg, J.M., Event and consequence vulnerability: effects on the disaster recovery process, http://www.udel.edu/DRC/preliminary/217.pdf Retrieved on 2007-07-26, 1993.

[16] Oliver-Smith, A., Success and failure in post-disaster settlement, Disasters, 15, pp. 12-24, 1991.

[17] Boyce, J.K., Let them eat risk? Wealth, rights and disaster vulnerability, Disaster, 24(3), pp. 254-261, 2000.

[18] McEntire, D.A., Correspondence, Disasters, 24(1), pp. 78-79, 1999.

[19] Bolton, P.A., Liebow, E.B. \& Olson, J.L., Community context and uncertainty following a damaging earthquake: low-income Latinos and Los Angeles, California. The Environmental Professional, 15, pp. 240-247, 1993. 
[20] Cooper, F. \& Laughy, L., Managing hazards in a changing multinational world, unpublished paper, 1994.

[21] Bolin, R. \& Bolton, P.A., Race, Religion, and Ethnicity in Disaster Recovery, Monograph No. 42, Institute of Behavioral Science, University of Colorado: Boulder, CO, 1986.

[22] Peacock, W.G., Killian, C.D. \& Bates, F.L., The effects of disaster damage and housing aid on household recovery following the 1976 Guatemalan earthquake. The International Journal of Mass Emergencies and Disasters, 5(1), pp. 63-88, 1987.

[23] World Health Organization, Gender, Women, and Health, http://www.searo.who.int/en/ Section13/Section390_8282.htm (accessed on June 12, 2008).

[24] Peacock, W., Hurricane Andrew: Ethnicity, Gender, and the Sociology of Disasters, Routledge: London, 1997.

[25] World Commission on Environment and Development, Our Common Future, Oxford University Press: New York, 1987.

[26] Monday, J.L., Building back better: creating a sustainable community after disaster. Natural Hazard Informer, 3, pp. 1-11, 2002.

[27] Natural Hazards Research and Applications Information Center, University of Colorado, Holistic Disaster Recovery: Ideas for Building Local Sustainability after a Natural Disaster, Public Entity Risk Institute (PERI): Fairfax, VA, 2006.

[28] Wu, J.Y. \& Lindell, M.K., Housing reconstruction after two major earthquakes: the 1994 Northridge earthquake in the United States and the 1999 Chi-Chi earthquake in Taiwan. Disasters 28(1), pp. 63-81, 2004.

[29] Alexander, D., Principles of Emergency Planning and Management. Oxford University Press: New York, 2002.

[30] Peacock, W.G. \& Girard, C., Ethnic and racial inequalities in hurricane damage and insurance settlements. Hurricane Andrew: Ethnicity, Gender and the Sociology of Disasters, eds W.G. Peacock, B.H. Morrow \& H. Gladwin, Routledge: London, pp. 171-190, 1997.

[31] Barnes, J.C., A Guide to Business Continuity Planning. Wiley: West Sussex, UK, 2001.

[32] Bell, J.K., Disaster Survival Planning: A Practical Guide for Businesses, Disaster Survival Planning, 1991.

[33] Goodin, R., The sustainability ethic: political, not just moral, Journal of Applied Philosophy, 16(3), pp. 247-254, 1999.

[34] Anderson, M.B. \& Woodrow, P.J., Rising from the Ashes: Development Strategies in Times of Disaster, Westview Press: Boulder, CO, 1989.

[35] Berke, P. \& Beatley, T., After the Hurricane: Linking Recovery to Sustainable Development, John Hopkins University Press: Baltimore, MD, 1997.

[36] Pearce, L., Disaster management and community planning, and public participation: how to achieve sustainable hazard mitigation" Natural Hazards, 28, pp. 211-228, 2003.

[37] Berke, P., Natural-hazard reduction and sustainable development: a global assessment. Journal of Planning Literature, 9(4), pp. 370-382, 1995.

[38] Sen, A., Development as capabilities expansion. Journal of Development Planning, 19, pp. 41-58, 1989.

[39] Sen, A., Capability and well-being. The Quality of Life, eds M. Nussbaum \& A. Sen, Clarendon Press: Oxford, UK, pp. 30-53, 1993.

[40] Sen, A., Development as Freedom, Anchor Books: New York, 1999.

[41] Sen, A., Commodities and Capabilities, Oxford University Press: New York, 1999.

[42] Sen, A., Elements of a theory of human rights. Philosophy \& Public Affairs, 32(4), pp. 315-356, 2004. 
[43] Nussbaum, M., Woman and Human Development: The Capabilities Approach, Cambridge University Press: Cambridge, UK, 2001.

[44] Nussbaum, M., Aristotle, politics, and human capabilities: a response to Antony, Arneson, Charlesworth, and Mulgan. Ethics, 111(1), pp. 102-140, 2000.

[45] Anand, S. \& Sen, A., The income component of the Human Development Index. Journal of Human Development, 1(1), pp. 83-106, 2000.

[46] Berges, S., Why the capability approach is justified. Journal of Applied Philosophy, 24(1), pp. 16-25, 2007.

[47] Raworth, K. \& Stewart, D., Critiques of the Human Development Index: a review. S. Fukuda-Parr \& A.K. Shiva Kumar Readings in Human Development, eds S. Fukuda-Parr \& A.K. Shiva Kumar, Oxford University Press: Oxford, UK, pp. 140-152, 2003.

[48] United Nation Development Program, Human Development Report website, http://hdr.undp. org/ Retrieved on 2007-07-26, 2007.

[49] Murphy, C. \& Gardoni, P., The role of society in engineering risk analysis: a capabilities-based approach. Risk Analysis, 26(4), pp. 1085-1095, 2006.

[50] Gardoni, P. \& Murphy, C., A capabilities-based approach to measuring the societal impacts of natural and man-made hazards. Natural Hazard Review, 2006 (submitted).

[51] Murphy, C. \& Gardoni, P., Determining public policy and resource allocation priorities for mitigating natural hazards: a capabilities-based approach. Science and Engineering Ethics, 13(4), pp. 489-504, 2007.

[52] Murphy, C. \& Gardoni, P., The acceptability and the tolerability of societal risks: a capabilitiesbased approach. Science and Engineering Ethics, 14(1), pp. 77-92, 2007. 